\title{
A Combined Microwave Imaging Algorithm for Localization and Moisture Level Estimation in Multilayered Media
}

\author{
Adel Omrani*, Rahul Yadav ${ }^{\ddagger}$, Guido Link*, Marko Vauhkonen ${ }^{\ddagger}$, Timo Lähivaara ${ }^{\ddagger}$, and John Jelonnek*† \\ ${ }^{*}$ Institute for Pulsed Power and Microwave Technology (IHM), Karlsruhe Institute of Technology (KIT), Karlsruhe, Germany \\ ${ }^{\dagger}$ Institute of Radio Frequency Engineering and Electronics (IHE), Karlsruhe Institute of Technology (KIT), Karlsruhe, Germany \\ ${ }_{\ddagger}^{\ddagger}$ Department of Applied Physics, University of Eastern Finland, Kuopio, Finland \\ Email: adel.hamzekalaei@kit.edu
}

\begin{abstract}
In this work, a multistatic uniform diffraction tomography (MUDT) method, that was proposed by the authors as a new qualitative imaging method just recently, is combined with the quantitative Bayesian inversion framework. In this combined approach, MUDT is applied to find the location of the moisture and this localization is employed as a pre-knowledge for the Bayesian framework to estimate the moisture levels in a polymer foam. The proposed combined algorithm might become a major part of the development of a new kind of intelligent industrial microwave drying systems. The imaging algorithm is tested with simulated measurement data. The frequency band from $8 \mathrm{GHz}$ to $12 \mathrm{GHz}$ (X-band) is used for the MUDT algorithm whereas a single frequency of $8.2 \mathrm{GHz}$ is assumed for the Bayesian framework. The first results demonstrate the ability of the developed combined algorithm for optimizing the computational load unlike seen in the quantitative inversion approaches.
\end{abstract}

Index Terms-industrial drying, inverse EM scattering, microwave imaging, MUDT, Bayesian inversion method

\section{INTRODUCTION}

Microwave drying is an emerging technology in the industry for batch and continuous processing due to the possibility of volumetric and selective heating. Intraction of high-power microwaves to materials with certain moisture content results in significant energy and time-saving as compared to conventional, convective, and radiative heating. Therefore, microwave based drying is used in a wide variety of applications, e.g. for dielectric heating of ceramics, food, chemical, and pharmaceutical industries [1].

We work on the HEPHAISTOS microwave system [2] that has a patented hexagonal cross-section design with an overall length and hexagon circumferential diameter of $4 \mathrm{~m}$ and $1 \mathrm{~m}$, respectively. It has a modular structure and consists of three microwave modules of the same type; each module is $1 \mathrm{~m}$ in length and mounted with six slotted waveguide antennas delivering a total power of $36 \mathrm{~kW}$ at the $2.45 \mathrm{GHz}$ ISMband. Another unique feature of the HEPHAISTOS microwave system is the conveyor belt that can be added and that allows a continuous drying process [3]. One target application is the processing of polymer foam that has low thermal conductivity. The objective is to dry the foam uniformly with the final target to keep the moisture under a certain level inside the complete volume at the end of the heating process. Oftenly those foams have non-uniform moisture distribution at the inlet. Together with a nonuniform distribution of the electromagnetic field that leads to the formation of hot-spots. Those hot spots might deform and destroy the foam respectively. Intelligent control of distributed microwave sources is a possibility that might efficiently address the non-uniform moisture distribution [3]. However, it requires the in-situ and non-invasive measurements of the unknown moisture distribution inside the material under test. Thus, to attain the moisture location and level inside the polymer foam in a running belt process, a microwave tomography (MWT) method is integrated into the HEPHAISTOS system.

The MWT imaging algorithm is used to estimate the moisture distribution and its level in the foam of infitite length and with a large cross-sectional size $(36 \mathrm{~cm} \times 8 \mathrm{~cm})$. A preferred method is to apply an optimization based quantitative reconstruction approach. However, for the mentioned large problem size, this approach suffers from heavy computational burden due to high number of unknowns. Here, pre-knowledge of the scatterer location (distribution) under a known background can be useful. As it can limit the unknowns only to the scatterer domain. Hence, improved estimates can be obtained with less computational burden.

In this study, the recently developed qualitative imaging algorithm MUDT is used for the first time to estimate the location of the scatterers. This estimation is provided as initial input to the iterative Bayesian algorithm [4]. The MUDT is an extended version of the uniform diffraction tomography (UDT). It can exploit all the scattering elements of the scattering matrix rather than only the diagonal elements as in UDT. Thus, results in better resolution than UDT. Also, under condition 1) the low number of antennas, and 2) if two adjacent antennas are not in close vicinity to each other, the UDT imaging algorithm fails to truly reconstruct the location of the scatterer [5]. With this combined approach, more accurate estimates can be obtained with improved computational time.

The paper is organized as follows: Section II provides a 

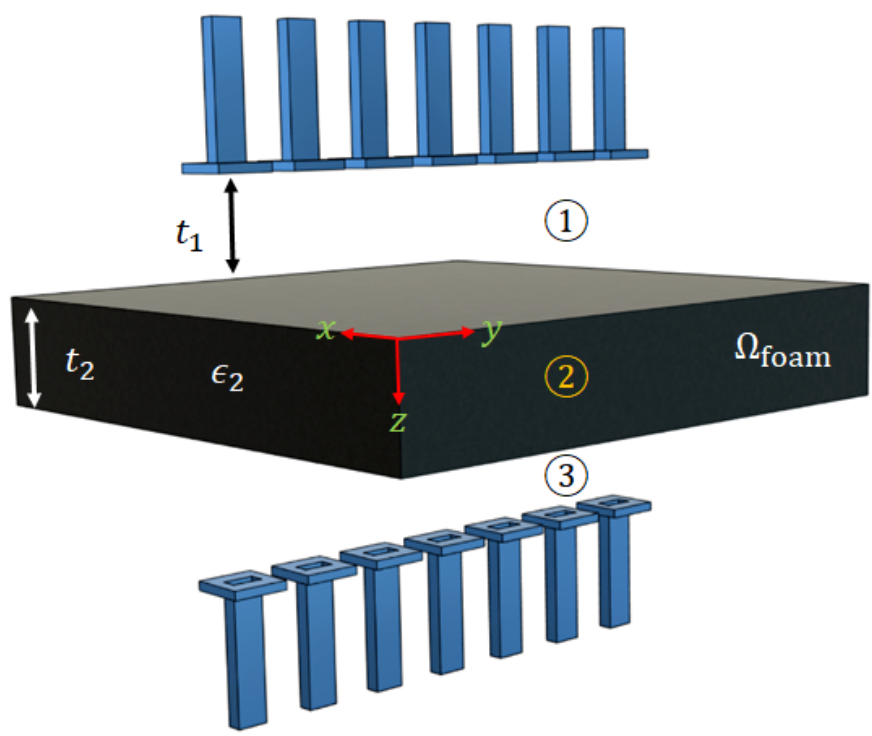

Fig. 1. 3-D MWT setup used in this study to generate synthetic data.

view over the geometrical configuration. Section III and Section IV details the MUDT qualitative method, and quantitative Bayesian inversion framework, respectively. In Section V the simulation results are presented and Section VI shows the discussion and concluding remarks.

\section{MWT SETUP}

The 3-D configuration of the multistatic microwave imaging system is illustrated in Fig. 1. Different to most of the known qualitative applications, where the antenna array is located on one side of the medium only (limited-angle) [6], [7], here, in order to determine the dielectric value, the antenna array is located at both sides of the foam (full-angle). The computational domain consist of the polymer foam of size $\Omega_{\text {foam }}=[-25,25] \times[-18,18] \times[-4,4] \mathrm{cm}$. It is placed in free space. The setup of the sensor consists of WR-90 waveguide antennas that are capable to excite an electromagnetic field into the medium at $8-12 \mathrm{GHz}$. An array of 7 waveguide antennas is located in semi-infinite free space, above and below the polymer foam. The distance of the antenna to the top of the polymer foam is $t_{1}=16 \mathrm{~cm}$ and the center to center distance between two adjacent antennas is $6 \mathrm{~cm}$. In free space, the relative dielectric constant is set to $\epsilon_{r}=1-0 j$ whereas the relative dielectric constant of the foam is set to $\epsilon_{2}$. It is assumed that the dielectric property of the foam varies is in z-direction only. The free space is represented as layer (1) and (3) and the foam is presented as layer (2).

\section{QUALITATIVE METHOD: MUDT}

In this section the two reconstruction approaches are discussed in more detail. The combined approach is evaluated for a fixed cross-section (i.e. $y \times z$ ) of the foam. As a result, the formulation is represented in 2-D only. That is done to simplify the approach. An expansion to full 3-D is possible.
Under the assumption of an excitation by a point source and under the assumption of antennas located at the top of the foam only, the scattered electric fields due to the irregularities in the layer (2), can be written in the following form [8], [9]

$$
\vec{E}_{2}^{\mathrm{sct}}\left(\vec{r}_{r}, \vec{r}_{t}\right)=k^{2} \int_{\Omega_{\mathrm{foam}}} \overline{\bar{G}}_{\mathrm{eb}}^{(21)}\left(\vec{r}_{r}, \vec{r}^{\prime}\right) \cdot O_{2}\left(\vec{r}^{\prime}\right) \vec{E}_{2}^{\mathrm{tot}}\left(\vec{r}^{\prime}\right) d \vec{r}^{\prime}
$$

where $\vec{E}_{2}^{\text {tot }}\left(\vec{r}^{\prime}\right)$ is the total electric field in layer (2) and $\vec{E}_{2}^{\text {sct }}\left(\vec{r}_{r}, \vec{r}_{t}\right)$ represents the scattered field due to the unknown irregularities in layer (2). $O_{2}\left(\vec{r}^{\prime}\right)=\left(\epsilon_{r}\left(\vec{r}^{\prime}\right)-\epsilon_{2}\right)$ is the object function. $\epsilon_{r}\left(\vec{r}^{\prime}\right)$ is the profile of the dielectric constant of the target and $\epsilon_{2}$ denotes the dielectric constant of the background. $\Omega_{\text {foam }} \subset \mathbb{R}^{2}$ is the region of interest which is layer (2) and and $\vec{r}^{\prime} \in \Omega_{\text {foam. }}$. Throughout the paper, $e^{-j \omega t}$ harmonic time convention is assumed and suppressed. $\omega$ is the angular frequency. In $(1)$, the vectors $\vec{r}_{r}=\left(y_{r}, z_{r}\right)$ and $\vec{r}_{t}=\left(y_{t}, z_{t}\right)$ represent observation and source points while $\overline{\bar{G}}_{\mathrm{eb}}^{(21)}\left(\vec{r}_{r}, \vec{r}_{t}\right)$ is the electric background (multilayered media without any scatterer inside) dyadic Green's function (DGF). The superscript (21) denotes that the source point is located in layer (1) and the observation point is in layer (2).

By applying the first-order Born approximation, the total electric field $\vec{E}_{2}^{\text {tot }}$ can be replaced by the background electric field of the layer. Due to the excitation by a point source the electric field can be replaced by the Green's function and also using the symmetry property of Green's function, a model for the scattering electric field can be expressed as follow

$$
E_{2}^{\mathrm{sct}}\left(\vec{r}_{r}, \vec{r}_{t}\right)=k^{2} \int_{\Omega_{\mathrm{foam}}} \overline{\bar{G}}_{\mathrm{eb}}^{(21)}\left(\vec{r}_{r}, \vec{r}^{\prime}\right) \cdot O_{2}\left(\vec{r}^{\prime}\right) \bar{G}_{\mathrm{eb}}^{(21)}\left(\vec{r}^{\prime}, \vec{r}_{t}\right) d \vec{r}^{\prime}
$$

The spectral representation of the Green's function in layer (2) (modeled by the incident field in that layer) when the point source is located in layer (1) is [10]

$G_{\mathrm{eb}}^{(21)}\left(\vec{r}, \vec{r}_{t}\right)=\frac{1}{\pi} \int_{-\infty}^{\infty} \tilde{T}_{2}\left(k_{y}, k_{z}\right) \frac{e^{-j k_{z 2}\left(z-z_{t}\right)}}{k_{z 1}} e^{-j k_{y}\left(y-y_{t}\right)} d k_{y}$

if $z>z_{t}$ and $\Im\left(k_{2}^{2}-k_{y}^{2}\right)^{\frac{1}{2}}<0 . \tilde{T}_{2}\left(k_{y}, k_{z}\right)$ is the transmission coefficient in layer (2) and can be obtained by applying the continuity conditions between layers for the transverse magnetic field in $x$-direction $\left(T M_{x}\right)$. The dispersion relation in the layer (l) $(l=1,2,3)$ is expressed by $k_{z l}=\sqrt{k_{l}^{2}-k_{y_{l}}^{2}}$ and $k_{l}=k_{0} \sqrt{\epsilon_{l}}$ is the wavenumber in layer (l) while $k_{0}$ is the free-space wavenumber.

From (2) the object function can be determined. Substituting (3) in (2) with the prime integrand for the second Green's term, changing variables to $k_{y}=k_{y}^{\prime}+k_{y}^{\prime \prime}$ and using 2-D spatial Fourier definition for the received signal and employing the stationary phase approximation method and finally after some straightforward calculation, the object function can be obtained as given in (4)(see next page), where $\tilde{E}_{2}^{\text {sct }}\left(k_{y}^{\prime \prime}, \omega\right)$ is the spatial Fourier transform of the received scattered field and where $\angle$ is the phase. 


$$
O_{2}(y, z)=\frac{\int_{\omega} \int_{-\infty}^{+\infty} \frac{1}{\left|\tilde{T}_{2}\right|^{2}} \frac{k_{2}}{\omega k_{z 2}^{\prime \prime}} e^{-j\left[k_{z 2}^{\prime \prime} z+\angle \tilde{T}_{2}\left(\frac{k^{\prime \prime}}{2}, k_{z 2}^{\prime \prime}, t_{l}\right)+\frac{k^{\prime \prime}}{2}\left(y_{t}-y_{r}\right)-\frac{\pi}{4}\right]} \tilde{E}_{2}^{\mathrm{sct}}\left(k_{y}^{\prime \prime}, \omega\right) e^{j k_{y}^{\prime \prime} y} d k_{y}^{\prime \prime} d \omega}{2 \pi \int_{\omega} \int_{-\infty}^{+\infty} \frac{k_{2}}{\omega k_{z 2}^{\prime \prime}} \frac{1}{\sqrt{\frac{k_{2}^{2}}{k_{z 2}^{3}} z+\frac{\partial^{2}}{\partial k_{y}^{2}}\left[\angle \tilde{T}_{2}\right]_{\left.\right|_{k_{y}} \frac{k^{\prime \prime}}{2}}}} d k_{y}^{\prime \prime} d \omega}
$$

The object function gives the location of the target, denoted by

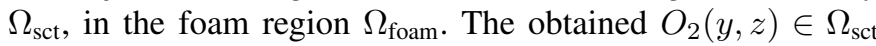
will be used as an initial updated domain for the Bayesian algorithm. So, instead of using $\Omega_{\text {foam }}$, by employing $\Omega_{\text {sct }}$, the computational burden of estimating the moisture level will decrease and algorithm may not trap in a local minimum. From here on the vector notations are omitted.

\section{QUANTITATIVE METHOD: BAYESIAN INVERSION FRAMEWORK}

Consider an inverse problem of identifying an unknown parameter $\epsilon_{r} \in \mathbb{C}$ given noisy measurement data $E^{\text {meas }} \in \mathbb{C}$ according to the observation model

$$
E^{\text {meas }}=\mathscr{F}\left(\epsilon_{r}\right)+\xi
$$

where $\mathscr{F}: \epsilon_{r} \rightarrow E^{\text {meas }}$ is the forward model represented by (1) that maps $\epsilon_{r}$ to the measurement, and $\xi$ denotes the additive measurement noise component in the antenna. The unknown parameter and noise terms are considered mutually independent. Note that the measurement data and unknown terms are complex quantity, therefore in the present study the real and imaginary parts are concatenated to solve the inverse problem.

In Bayesian framework the unknown parameters are treated as random variables, and information about them are expressed in terms of probability densities. The inverse problem is then expressed as given the measured scattering data, the task is to find the conditional probability density $\pi\left(\epsilon_{r} \mid E^{\mathrm{sct}}\right)$ for the unknown quantity $\epsilon_{r}$. The conditional probability density is constructed using the Bayes' formula as

$$
\pi\left(\epsilon_{r} \mid E^{\text {meas }}\right)=\frac{\pi\left(E^{\text {meas }} \mid \epsilon_{r}\right) \pi\left(\epsilon_{r}\right)}{\pi\left(E^{\text {meas }}\right)} \propto \pi\left(E^{\text {meas }} \mid \epsilon_{r}\right) \pi\left(\epsilon_{r}\right),
$$

where $\pi\left(\epsilon_{r} \mid E^{\text {meas }}\right)$ is the posterior density, $\pi\left(E^{\text {meas }} \mid \epsilon_{r}\right)$ is likelihood density which represents the distribution of the measured data if $\epsilon_{r}$ is known, and $\pi\left(\epsilon_{r}\right)$ is the prior density which contains the prior information available for unknown $\epsilon_{r}$. The denominator is the marginal density of the measured data and plays the role of normalization constant and is often ignored.

The posterior density in (6) contains the complete solution of the inverse problem in the Bayesian framework and can be expressed by point estimates. One of the most common point estimate in tomographic imaging problems is the maximum a posteriori (MAP). The MAP estimates can be computed from the posterior as

$$
\widehat{\epsilon}_{r_{\mathrm{MAP}}}=\arg \max _{\epsilon_{r}} \pi\left(\epsilon_{r} \mid E^{\text {meas }}\right) .
$$

This problem is equivalent to the minimization problem [4]

$$
\widehat{\epsilon}_{r_{\mathrm{MAP}}}=\arg \min _{\epsilon_{r}}\left\{\left\|L_{\xi}\left(E^{\text {meas }}-\mathscr{F}\left(\epsilon_{r}\right)\right)\right\|^{2}+\left\|L_{\epsilon_{r}}\left(\epsilon_{r}-\eta_{\epsilon_{r}}\right)\right\|^{2}\right\} \text {, }
$$

which is a regularized non-linear least-squares problem. In (8), $L_{\xi}$ is the Cholesky factor of the inverse of the noise covariance matrix $\Gamma_{\xi} ; L_{\epsilon_{r}}$ is a Cholesky factor of the inverse of the prior covariance matrix $\Gamma_{\epsilon_{r}}$ and $\eta_{\epsilon_{r}}$ denotes the mean value of the prior. This minimization problem can be formally solved using Gauss-Newton method as

$$
\epsilon_{r_{i+1}}=\epsilon_{r_{i}}+\alpha_{i} A^{-1} B
$$

with,

$$
\begin{gathered}
A=\left(J_{i}^{T} \Gamma_{\xi}^{-1} J_{i}+\Gamma_{\epsilon_{r}}^{-1}\right) \\
B=\left(J_{i}^{T} \Gamma_{\xi}^{-1}\left(E^{\text {meas }}-\mathscr{F}\left(\epsilon_{r}\right)\right)-\Gamma_{\epsilon_{r}}^{-1}\left(\epsilon_{r_{i}}-\eta_{\epsilon_{r}}\right)\right)
\end{gathered}
$$

where $\alpha_{i}$ is the step length parameter, $J_{i}$ is a Jacobian matrix, and index $i$ is the iteration number.

An initial guess value is required to compute (9). A close initial guess to the true value can result in faster convergence. Hence, here the initial dielectric value $\epsilon_{r_{0}}$ is supplied by the MUDT imaging algorithm. To the MUDT reconstruction, obtained using (4), firstly a threshold value $\wp$ is applied to retrieve the shape of the scatterer

$$
O_{\text {threshold }}=\left.O_{2}(y, z)\right|_{>\wp} \text {. }
$$

The choice of the threshold value is somewhat heuristic. After the thresholding operation, the moisture area and dry part can be assigned to dielectric constant values as

$$
\epsilon_{r_{0}}= \begin{cases}\epsilon_{\text {moisture }}, & \forall O_{\text {threshold }} \in \Omega_{\text {sct }} \\ \epsilon_{\text {dry }}, & \forall O_{\text {threshold }} \notin \Omega_{\text {sct }}\end{cases}
$$

where $\epsilon_{\text {moisture }}$ denotes the moisture areas in the target domain $\Omega_{\text {sct }}$ and $\epsilon_{\text {dry }}$ denotes the dry areas in the foam. 

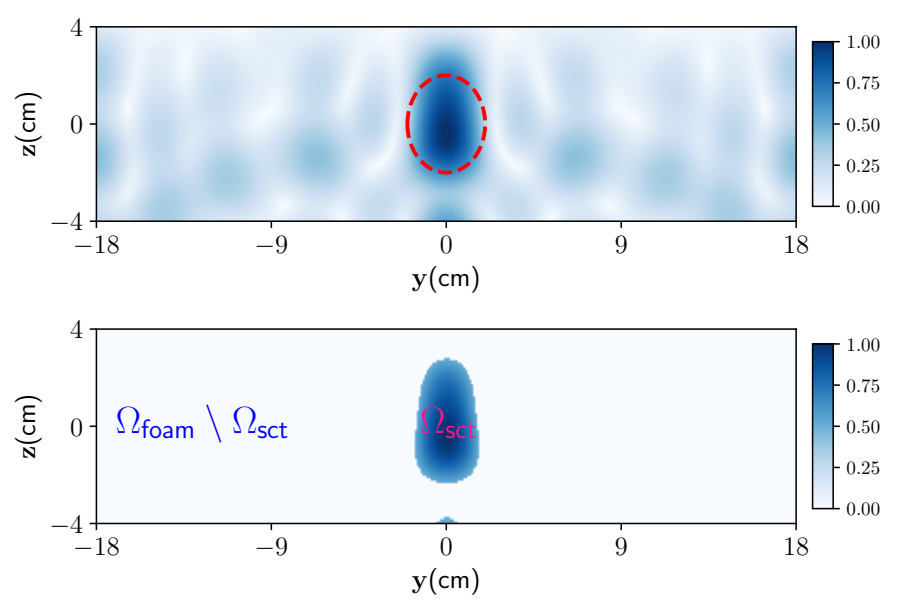

Fig. 2. Localization from MUDT with the true scatterer shape in red color is shown in top. The bottom image shows the effect of thresholding operation.

\section{RECONSTRUCTION}

In this section, we investigate the proposed combined microwave imaging algorithm. To generate the simulated data, the 3D time domain solver of the commercial Software CST Studio Suite is used. The data is stored in terms of scattering matrix of size $14 \times 14$. Moisture is given a spherical shape with $\epsilon_{r}=1.7-0.1 j$ and surrounded by the dry part with $\epsilon_{2}=1.16-0.01 j$. These dielectric constant values are equivalent to $40 \%$ and $0 \%$ wet basis moisture levels (determined based on the cavity-perturbation method carried out at the HEPHAISTOS laboratory at the KIT). The implementation details are given as follows.

\section{A. MUDT}

To obtain the qualitative image using the MUDT, only the scattering parameters of the top antennas are used. Moreover, in addition to the diagonal element of the scattering matrix, the $S_{i(i+1)},(i=1,2, . ., 6)$ of the top antennas are used as well for the image reconstruction. Fig. 2(top) shows the reconstructed image using the MUDT imaging algorithm. As can be perceived from this figure, the location of the scatterer is truly obtained with normalized value of the object function. In the next step, a threshold operation is applied where the values more than 0.6 (equivalent to the scatterer location) are set to $\epsilon_{\text {moisture }}$ and rest areas are set to the dielectric constant of the background $\epsilon_{\text {dry }}$ as can be seen in right image of Fig. 2(bottom).

\section{B. Bayesian inversion with MUDT}

Here, the full matrix of simulated data for $8.2 \mathrm{GHz}$ is used. It should be noted that we used the symmetry between the S-parameters to fill out the scattering matrix. To compute (9), lower limit of X-band frequency is chosen to have less number of unknowns in the inverse problem. The scattered electric field is calculated using method of moment (MoM) method with pulse basis and point-matching testing function. The antennas are modeled as point source, and electric field data is converted to the scattering matrix with a calibration scheme, also Jacobian matrix is evaluated [11]. The noise and prior covariance matrix is calculated as follows.

1) Noise model: Let us denote the noise variance of the real and imaginary part of the complex-valued scattered field data to be $\sigma_{\mathbb{R}}$ and $\sigma_{\mathbb{I}}$, respectively. The noise covariance is then given as,

$$
\Gamma_{\xi}=\left[\begin{array}{cc}
\sigma_{\mathbb{R}}^{2} \mathbf{I}_{n \times n} & 0_{n \times n} \\
0_{n \times n} & \sigma_{\mathbb{I}}^{2} \mathbf{I}_{n \times n}
\end{array}\right],
$$

where $\mathrm{I}$ is an identity matrix and $n$ denotes the total number of measurements. The noise level may be estimated from repeated measurements, but for this case of simulation data, a small noise level is assumed. The standard deviation of the real and imaginary part are set $10^{-4}$.

2) Prior model: The moisture field variation inside the foam is assumed to be smooth. Here, such a random field is generated using a multivariate Gaussian distribution with anisotropic covariance structure $C$ [12], [13].

If real and imaginary part of the dielectric constant are assumed uncorrelated, then the prior covariance matrix can be written as

$$
\Gamma_{\epsilon_{r}}=\left[\begin{array}{cc}
\sigma_{\epsilon_{r}^{\prime}}^{2} C & 0 \\
0 & \sigma_{\epsilon_{r}^{\prime \prime}}^{2} C
\end{array}\right] .
$$

Here $\sigma_{\epsilon_{r}^{\prime}}$, and $\sigma_{\epsilon_{r}^{\prime \prime}}$ are the standard deviations for the real and imaginary parts of dielectric constant, respectively. For the standard deviations, the values of $\sigma_{\epsilon_{r}^{\prime}}=1$, and $\sigma_{\epsilon_{r}^{\prime \prime}}=0.05$ are set in all cases, respectively. The mean value of the prior $\eta_{\epsilon_{r}}$ is set to dielectric constant of the dry foam i.e $1.16-0.01 j$.

MUDT reconstructed dielectric values are used as initial value for iteration. Only the unknowns inside the moisture domain are solved and rest of the values are assumed dry in each iteration. As a stopping criteria, posterior norm in current and past iteration is compared. The estimation result using Bayesian approach with initial guess input from MUDT is depicted in Fig. 3. The real part of the dielectric constant is closely estimated. For the Bayesian approach the scatterer

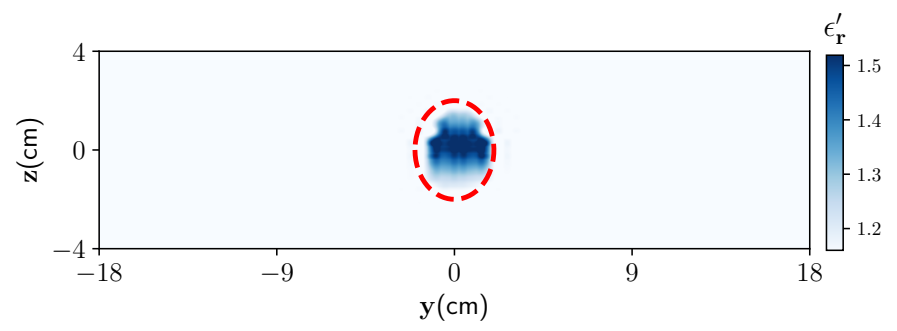

Fig. 3. MAP estimate using the proposed combined scheme with true shape of scatterer highlighted in red.

shape is not retrieved. This is dominantly caused due to smoothness assumption in the prior and modelling errors. Moisture distribution in such strict shape is hard to find in real scenario. Thus, smoothness prior assumption is sufficient. 


\section{CONCLUSION}

In this paper, MUDT and Bayesian inversion approaches are combined as a new imaging algorithm and tested on the simulated data to estimate the moisture content distribution and its level in a porous foam. The combined approach estimated the moisture scenario with optimized computational load. This approach is expected to overcome the computational challenges of conventional quantitative approaches when applied to solve the present problem. In the next step we investigate to assign the proper moisture levels for multiple scatterers in MUDT. In the final phase, the algorithm will be tested in a real scenario.

\section{ACKNOWLEDGMENT}

This work has been supported by the European Union's Horizon 2020 Research and Innovation Program under the Marie Skłodowska-Curie grant agreement No. 764902. (TOMOCON-www.tomocon.eu). The work is also supported by the Academy of Finland (Finnish Centre of Excellence of Inverse Modelling and Imaging, project number 312344, and project number 321761).

\section{REFERENCES}

[1] P. Rattanadecho and N. Makul, "Microwave-assisted drying: A review of the state-of-the-art," Drying Technology, vol. 34, no. 1, pp. 1-38, 2016.

[2] G. Link et al., "Faserverbund-leichtbau mit automatisierter mikrowellenprozesstechnik hoher energieeffizienz (flame) : Schlussbericht des bmbfverbundprojektes (kit scientific reports ; 7701)," tech. rep., Karlsruher Institut für Technologie (KIT), 2015. 34.12.01; LK 01.
[3] Y. Sun, Adaptive and Intelligent Temperature Control of Microwave Heating Systems with Multiple Sources. KIT Scientific Publishing, Karlsruhe, 2016.

[4] J. Kaipio and E. Somersalo, Statistical and Computational Inverse Problems. Springer-Verlag, 2005.

[5] A. Omrani, G. Link, and J. Jelonnek, "A multistatic uniform diffraction tomographic algorithm for real-time moisture detection," Unpublished.

[6] S. M. Moghadasi, M. Dehmollaian, and J. Rashed-Mohassel, "Time reversal imaging of deeply buried targets under moderately rough surfaces using approximate transmitted fields," IEEE Transactions on Geoscience and Remote Sensing, vol. 53, no. 7, pp. 3897-3905, 2015.

[7] V. Khorashadi-Zadeh and M. Dehmollaian, "Through a cinder block wall refocusing using sar back projection method," IEEE Transactions on Antennas and Propagation, vol. 67, no. 2, pp. 1212-1222, 2019.

[8] C. T. Tai, Dyadic Green's functions in electromagnetic theory. IEEE Press, 1994.

[9] A. Omrani, M. Moghadasi, and M. Dehmollaian, "Localisation and permittivity extraction of an embedded cylinder using decomposition of the time reversal operator," IET Microwaves, Antennas Propagation, vol. 14, pp. 851-859(8), July 2020.

[10] W. C. Chew, Waves and Fields in Inhomogenous Media. IEEE Press, 1995.

[11] A. Franchois and C. Pichot, "Microwave imaging-complex permittivity reconstruction with a Levenberg-Marquardt method," IEEE Transactions on Antennas and Propagation, vol. 45, no. 2, pp. 203-215, 1997.

[12] T. Lähivaara, R. Yadav, G. Link, and M. Vauhkonen, "Estimation of moisture content distribution in porous foam using microwave tomography with neural networks," IEEE Transactions on Computational Imaging, vol. 6, pp. 1351-1361, 2020.

[13] C. Rasmussen and C. Williams, Gaussian Processes for Machine Learning. The MIT Press, 2006. 


\section{Repository KITopen}

Dies ist ein Postprint/begutachtetes Manuskript.

\section{Empfohlene Zitierung:}

Omrani, A.; Yadav, R.; Link, G.; Vauhkonen, M.; Lahivaara, T.; Jelonnek, J.

A Combined Microwave Imaging Algorithm for Localization and Moisture Level Estimation in Multilayered Media.

2021. 15th European Conference on Antennas and Propagation (EuCAP),

22-26 March 2021, Düsseldorf

doi: $10.554 / I R / 1000133456$

Zitierung der Originalveröffentlichung:

Omrani, A.; Yadav, R.; Link, G.; Vauhkonen, M.; Lahivaara, T.; Jelonnek, J.

A Combined Microwave Imaging Algorithm for Localization and Moisture Level Estimation in Multilayered Media.

2021. 15th European Conference on Antennas and Propagation (EuCAP), 22-26 March 2021, Düsseldorf, Germany, Institute of Electrical and Electronics Engineers (IEEE). doi:10.23919/EuCAP51087.2021.9411327

\section{Lizenzinformationen: KITopen-Lizenz}

